\title{
Different methods of estimation for the one-parameter Akash distribution
}

\author{
Kadir KARAKAYA ${ }^{1, *}$ (D), Caner TANIŞ ${ }^{2}$ \\ ${ }^{l}$ Selcuk University, Department of Statistics, Konya/ TURKEY \\ ${ }^{2}$ Çankırı Karatekin University, Department of Statistics, Çankırl/TURKEY
}

\begin{abstract}
Akash distribution is a mixture of an exponential distribution and a gamma distribution with certain mixing proportions. Although the maximum likelihood estimation method has been proposed for the Akash distribution, there is no comprehensive comparison of different methods of estimation in the literature. This study provides five different methods of estimation, such as maximum likelihood, least-squares, weighted least-squares, AndersonDarling, and Crámer-von-Mises for Akash distribution. We consider a comprehensive Monte Carlo simulation study to compare the performances of these methods via the biases and meansquared errors of these estimators. Also, a real data application is performed.
\end{abstract}

\author{
Article info \\ History: \\ Received: 07.07.2020 \\ Accepted: 28.10.2020 \\ Keywords: \\ Akash distribution, \\ Maximum likelihood \\ method, Least-squares \\ method, Weighted \\ least-squares method, \\ Crámer-von-Mises \\ method.
}

\section{Introduction}

Akash distribution was introduced by Shanker [1]. It is a two-component mixture of exponential $(\theta)$ and gamma $(3, \theta)$ with their mixing proportions $\frac{\theta^{2}}{\theta^{2}+2}$ and $\frac{2}{\theta^{2}+2}$ respectively. The probability density function (pdf) and cumulative density function (cdf) of Akash distribution are, respectively, given by

$f(x ; \theta)=\frac{\theta^{3}}{\theta^{2}+2}\left(1+x^{2}\right) e^{-\theta x}, I_{(0, \infty)}(x)$

and

$$
F(x ; \theta)=1-\left[1+\frac{\theta x(\theta x+2)}{\theta^{2}+2}\right] e^{-\theta x},
$$

where $\theta>0$ is a scale parameter and $I_{(0, \infty)}(x)$ is an indicator function. Shanker [1] provided some characteristic properties of Akash distribution. It is examined the shape of the hazard function with its graphs and stated that Akash distribution is more flexible than Lindley and exponential distribution since its hazard function can be increasing and decreasing shape in [1]. Akash distribution is taken the attention of many authors recently. Some of these studies are listed as follows: Shanker and Fesshaye [2] considered a comprehensive study, including real data applications, to compare the potentials of fitted distributions for modeling lifetime data. They chose Shanker [3], Lindley [4], and exponential distributions as competitor distributions for Akash distribution in modeling real data. Shanker and Shukla [5] studied two parameters Akash distribution. Shanker et al. [6] suggested a generalization of Akash distribution. Shanker et al. [7] proposed a new distribution called Poisson-Akash distribution. The method of moments and maximum likelihood methods are also discussed to estimate the parameters of this model in [7]. Ganaie and Rajagopalan [8] described a new three parameters distribution by weighting on the Akash distribution.

Although many authors used Akash distribution to produce a new distribution, most of them did not tackle the problem of point estimation. The parameter estimation problem is one of the most popular topics that many authors have been dealing with recently. Estimation of the parameter is very important for distribution to obtain information about the unknown of the mass. Different methods can be used for parameter estimation. The maximum likelihood method is well-known for parameter estimation. 
However, alternative methods to the maximum likelihood method are used by many authors in the decade. In this case, they aim to compare different parameter estimation methods and determine which method is suitable for the parameter estimation of the relevant distribution. Some studies comparing different methods of estimation in recent years can be listed as follows: Gupta and Kundu [9] studied different estimation methods for the generalized exponential distribution. Kundu and Raqab [10] described a lifetime distribution called generalized Rayleigh. It also discussed different estimation methods for this distribution in [10]. Mazucheli et al. [11] compared different estimation methods for weighted Lindley distribution. Two parameters Rayleigh distribution was examined by Dey et al. [12] in terms of different methods of estimation. It was assessed those different estimation methods of Weibull distribution in [13]. Dey et al. [14] considered a comparison of methods of estimation for Nadarajah and Haghighi distribution.

This study aims to estimate the parameter of the Akash distribution with five different methods. For this reason, maximum likelihood estimator (MLE), leastsquare estimator (LSE), weighted least square estimator (WLSE), Anderson-Darling estimator (ADE), and Crámer-von-Mises estimator (CvME) are considered for point estimation.

This paper is organized as follows. Section 2 introduces five different methods of estimation. A comprehensive Monte Carlo simulation study is presented to evaluate the performances of these estimators according to bias and mean square error (MSE) criteria in Section 3. In Section 4, we consider

$$
\begin{aligned}
& Q_{L S}(\theta)=\sum_{i=1}^{n}\left(\left\{1-\left(1+\frac{\theta x_{(i)}\left(\theta x_{(i)}+2\right)}{\theta^{2}+2}\right) \exp \left(-\theta x_{(i)}\right)\right\}-\frac{i}{n+1}\right)^{2}, \\
& Q_{W L S}(\theta)=\sum_{i=1}^{n} \frac{(n+2)(n+1)^{2}}{i(n-i+1)}\left(\left\{1-\left(1+\frac{\theta x_{(i)}\left(\theta x_{(i)}+2\right)}{\theta^{2}+2}\right) \exp \left(-\theta x_{(i)}\right)\right\}-\frac{i}{n+1}\right)^{2}, \\
& Q_{C v M}(\theta)=\frac{1}{12 n}+\sum_{i=1}^{n}\left(\left\{1-\left(1+\frac{\theta x_{(i)}\left(\theta x_{(i)}+2\right)}{\theta^{2}+2}\right) \exp \left(-\theta x_{(i)}\right)\right\}-\frac{2 i-1}{2 n}\right)^{2}
\end{aligned}
$$

and a real data illustration. Finally, concluding remarks are given in Section 5.

\section{Point estimation on distribution parameter}

In this section, we examine five estimators for estimating the unknown parameter of $\operatorname{Akash}(\theta)$ distribution. We investigate maximum likelihood, least-squares, weighted least squares, Crámer-vonMises, and Anderson-Darling methods of estimation. Let $X_{1}, X_{2}, \ldots, X_{n}$ be a random sample from the Akash ( $\theta)$ distribution and $X_{(1)}<X_{(2)}<\ldots<X_{(n)}$ represent the corresponding order statistics. Additionally, $x_{(i)}$ denotes the observed value of $X_{(i)}$. Based on this information, the likelihood and log-likelihood function of the Akash $(\theta)$ distribution are given, respectively, by

$$
L(\theta)=\prod_{i=1}^{n} \frac{\theta^{3}}{\theta^{2}+2}\left(1+x_{i}^{2}\right) \exp \left(-\theta x_{i}\right)
$$

and

$$
\ell(\theta)=3 n \log (\theta)-n \log \left(\theta^{2}+2\right)+\sum_{i=1}^{n} \log \left(1+x_{i}^{2}\right)-\theta \sum_{i=1}^{n} x_{i} .
$$

Then, MLE of $\theta$ is given by

$$
\hat{\theta}_{1}=\underset{\theta}{\arg \max }\{\ell(\theta)\} \text {. }
$$

Let us define the following four functions which are used to obtain the different type of estimates: 


$$
\begin{aligned}
Q_{A D}(\theta) & =-n-\frac{1}{n} \sum_{i=1}^{n}\left((2 i-1) \log \left(\left\{1-\left(1+\frac{\theta x_{(i)}\left(\theta x_{(i)}+2\right)}{\theta^{2}+2}\right) \exp \left(-\theta x_{(i)}\right)\right\}\right)\right) \\
& +\frac{1}{n} \sum_{i=1}^{n}\left(\log \left(\left\{\left(1+\frac{\theta x_{(i)}\left(\theta x_{(i)}+2\right)}{\theta^{2}+2}\right) \exp \left(-\theta x_{(i)}\right)\right\}\right) .\right.
\end{aligned}
$$

the LSEs, WLSEs, CvMEs, and ADEs of the parameter $\theta$ are given, respectively, by

$\hat{\theta}_{2}=\underset{\theta}{\arg \min }\left\{Q_{L S}(\theta)\right\}$,

$\hat{\theta}_{3}=\underset{\theta}{\arg \min }\left\{Q_{W L S}(\theta)\right\}$,

$\hat{\theta}_{4}=\underset{\theta}{\arg \min }\left\{Q_{C v M}(\theta)\right\}$,

$\hat{\theta}_{5}=\underset{\theta}{\arg \min }\left\{Q_{A D}(\theta)\right\}$.

All estimates given in (5)-(9) can be achieved by optim function in R with BFGS algorithm.

\section{Simulation Study}

Table 1: Average biases of all estimates

\begin{tabular}{|c|c|c|c|c|c|c|}
\hline$\theta$ & $\mathrm{n}$ & MLE & LSE & WLSE & ADE & CVME \\
\hline 0.3 & 25 & 0.0038 & 0.0024 & 0.0022 & 0.0022 & 0.0030 \\
\hline & 50 & 0.0018 & 0.0011 & 0.0011 & 0.0010 & 0.0014 \\
\hline & 100 & 0.0009 & 0.0005 & 0.0005 & 0.0005 & 0.0007 \\
\hline & 250 & 0.0003 & 0.0002 & 0.0002 & 0.0001 & 0.0002 \\
\hline & & & & & & \\
\hline 0.7 & 25 & 0.0087 & 0.0055 & 0.0051 & 0.0050 & 0.0069 \\
\hline & 50 & 0.0041 & 0.0023 & 0.0023 & 0.0022 & 0.0031 \\
\hline & 100 & 0.0022 & 0.0012 & 0.0013 & 0.0012 & 0.0016 \\
\hline & 250 & 0.0008 & 0.0005 & 0.0005 & 0.0004 & 0.0006 \\
\hline & & & & & & \\
\hline 1.3 & 25 & 0.0191 & 0.0122 & 0.0111 & 0.0111 & 0.0159 \\
\hline & 50 & 0.0094 & 0.0058 & 0.0056 & 0.0054 & 0.0077 \\
\hline & 100 & 0.0048 & 0.0031 & 0.0031 & 0.0029 & 0.0040 \\
\hline & 250 & 0.0020 & 0.0013 & 0.0013 & 0.0012 & 0.0017 \\
\hline & & & & & & \\
\hline 2.2 & 25 & 0.0494 & 0.0347 & 0.0314 & 0.0305 & 0.0445 \\
\hline & 50 & 0.0252 & 0.0181 & 0.0170 & 0.0164 & 0.0230 \\
\hline & 100 & 0.0122 & 0.0085 & 0.0084 & 0.0078 & 0.0109 \\
\hline & 250 & 0.0052 & 0.0038 & 0.0039 & 0.0035 & 0.0048 \\
\hline
\end{tabular}

In this section, we consider an extensive Monte Carlo simulation study to evaluate the biases and MSEs of MLEs, LSEs, WLSEs, CvMEs, and ADEs of distribution parameter are estimated via 100000 trials. The acceptance-rejection algorithm is used to generate the data from $\operatorname{Akash}(\theta)$ distribution. BFGS algorithm is performed to get the five estimates given in (5)-(9). In Tables 1-2, biases and MSEs of MLE, LSE, WLSE, CVME, and ADE are reported for the true parameter $\theta=0.3,0.7,1.3,2.2$. The sample size $n=25,50,100,250$ is considered in the simulation study. Tables 1-2 shows that the biases and MSEs of all estimators are close to zero when the sample of size increases. 
Table 2: Average MSEs of the estimates

\begin{tabular}{|c|c|c|c|c|c|c|}
\hline$\theta$ & $\mathrm{n}$ & MLE & LSE & WLSE & ADE & CVME \\
\hline 0.3 & 25 & 0.0012 & 0.0014 & 0.0014 & 0.0013 & 0.0014 \\
\hline & 50 & 0.0006 & 0.0007 & 0.0007 & 0.0006 & 0.0007 \\
\hline & 100 & 0.0003 & 0.0003 & 0.0003 & 0.0003 & 0.0003 \\
\hline & 250 & 0.0001 & 0.0001 & 0.0001 & 0.0001 & 0.0001 \\
\hline & & & & & & \\
\hline 0.7 & 25 & 0.0064 & 0.0073 & 0.0069 & 0.0067 & 0.0073 \\
\hline & 50 & 0.0031 & 0.0036 & 0.0033 & 0.0033 & 0.0036 \\
\hline & 100 & 0.0015 & 0.0018 & 0.0017 & 0.0016 & 0.0018 \\
\hline & 250 & 0.0006 & 0.0007 & 0.0007 & 0.0007 & 0.0007 \\
\hline & & & & & & \\
\hline & 25 & 0.0242 & 0.0283 & 0.0266 & 0.0256 & 0.0286 \\
\hline & 50 & 0.0115 & 0.0134 & 0.0126 & 0.0123 & 0.0135 \\
\hline & 100 & 0.0056 & 0.0066 & 0.0062 & 0.0061 & 0.0066 \\
\hline & 250 & 0.0022 & 0.0026 & 0.0024 & 0.0024 & 0.0026 \\
\hline & & & & & & \\
\hline & 25 & 0.0946 & 0.1193 & 0.1097 & 0.1026 & 0.1213 \\
\hline & 50 & 0.0438 & 0.0547 & 0.0501 & 0.0488 & 0.0552 \\
\hline & 100 & 0.0210 & 0.0263 & 0.0240 & 0.0236 & 0.0264 \\
\hline & 250 & 0.0081 & 0.0102 & 0.0093 & 0.0092 & 0.0102 \\
\hline
\end{tabular}

\section{Real Data Applications}

In this section, a real data application of the Akash distribution is presented. For the comparison, we consider some one-parameter distributions such as exponential (E), Lindley (L), Shanker ( $\mathrm{SH}$ ) introduced by Shanker [3], Sujatha (SJ) introduced by Shanker [15]. The pdf of these distributions are given by

$$
\begin{aligned}
f_{E}(x) & =\theta \exp (-\theta x) \\
f_{L}(x) & =\frac{\theta^{2}}{\theta+1}(1+x) \exp (-\theta x) \\
\mathrm{f}_{\mathrm{SH}}(x) & =\frac{\theta^{2}}{\theta^{2}+1}(\theta+x) \exp (-\theta x) \\
\mathrm{f}_{\mathrm{SJ}}(x) & =\frac{\theta^{2}}{\theta^{2}+\theta+2}\left(1+x+x^{2}\right) \exp (-\theta x) .
\end{aligned}
$$

Five distributions are fitted to the real data set with the likelihood principle. The MLEs of all distributions parameter are also obtained by the BFGS algorithm. The MLEs of parameters and related standard errors for Akash, E, L, SH, and SJ distributions are reported in Table 3. The log-likelihood $(\ell),-2 \ell$, Akaike's information criterion (AIC), Bayesian information criterion (BIC), corrected Akaike' s information criterion (AICc), Hannan Quinn information criterion
(HQIC), Kolmogorov-Smirnov statistic (KS), Anderson-Darling statistic(AD), Cramer von Mises statistic $(\mathrm{CvM})$ and related p-values are obtained as Table 3. Also, LSEs, WLSEs, CvMEs, and ADEs of the parameter of the fitted distributions are reported in Table 3. Table 3 shows that the Akash distribution is the best-fitted model considering all criterion and statistics for failure time data. Figures 1-2 illustrates the fitted cdfs and pdfs of Akash distribution for five methods of estimation.

The data set is given by Murthy et al. [16] and represents the failure time of 20 components. The data are $0.072,4.763,8.663,12.089,0.477,5.284,9.511$, 13.036, 1.592, 7.709, 10.636, 13.949, 2.475, 7.867, $10.729,16.169,3.597,8.661,11.501$ and 19.809 . 
Table 3: Real data analysis results for the failure time data

\begin{tabular}{llllll}
\hline & Akash & $\mathrm{E}$ & $\mathrm{L}$ & $\mathrm{SH}$ & $\mathrm{SJ}$ \\
\hline$\ell$ & -61.6744 & -62.6346 & -61.3792 & -62.2797 & -61.8345 \\
$-2 \ell$ & 123.3488 & 125.2693 & 122.7583 & 124.5595 & 123.6689 \\
$\mathrm{AIC}$ & 125.3488 & 127.2693 & 124.7583 & 126.5595 & 125.6689 \\
BIC & 126.3445 & 128.2650 & 125.7541 & 127.5552 & 126.6647 \\
CAIC & 125.5710 & 127.4915 & 124.9805 & 126.7817 & 125.8912 \\
HQIC & 125.5431 & 127.4636 & 124.9527 & 126.7538 & 125.8633 \\
KS & 0.1663 & 0.2493 & 0.2022 & 0.2065 & 0.1707 \\
AD & 0.8635 & 1.1600 & 0.6904 & 0.8684 & 0.8410 \\
CvM & 0.0978 & 0.2349 & 0.1140 & 0.1256 & 0.0953 \\
KS $p$ value & 0.5807 & 0.1397 & 0.3398 & 0.3163 & 0.5482 \\
AD $p$ value & 0.4359 & 0.2827 & 0.5649 & 0.4327 & 0.4508 \\
CvM $p$ value & 0.6004 & 0.2094 & 0.5244 & 0.4766 & 0.6132 \\
$\hat{\theta}_{1}$ & 0.3427 & 0.1186 & 0.2162 & 0.2352 & 0.3293 \\
$\hat{\theta}_{2}$ & 0.3150 & 0.0954 & 0.1930 & 0.2067 & 0.3026 \\
$\hat{\theta}_{3}$ & 0.3192 & 0.1041 & 0.2002 & 0.2133 & 0.3073 \\
$\hat{\theta}_{4}$ & 0.3309 & 0.1003 & 0.2019 & 0.2179 & 0.3174 \\
$\hat{\theta}_{5}$ & 0.3171 & 0.0964 & 0.1945 & 0.2083 & 0.3047 \\
\hline
\end{tabular}

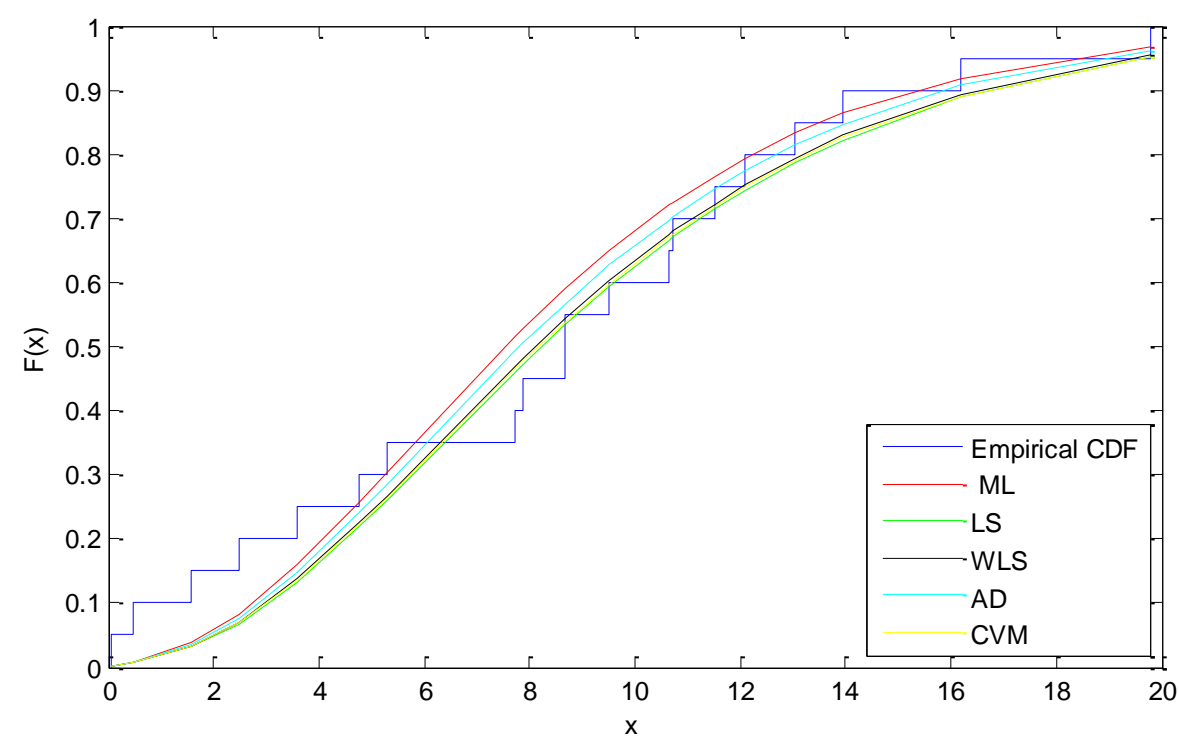

Figure 1. The fitted cdfs of Akash distribution for different methods of estimation based on failure time data 


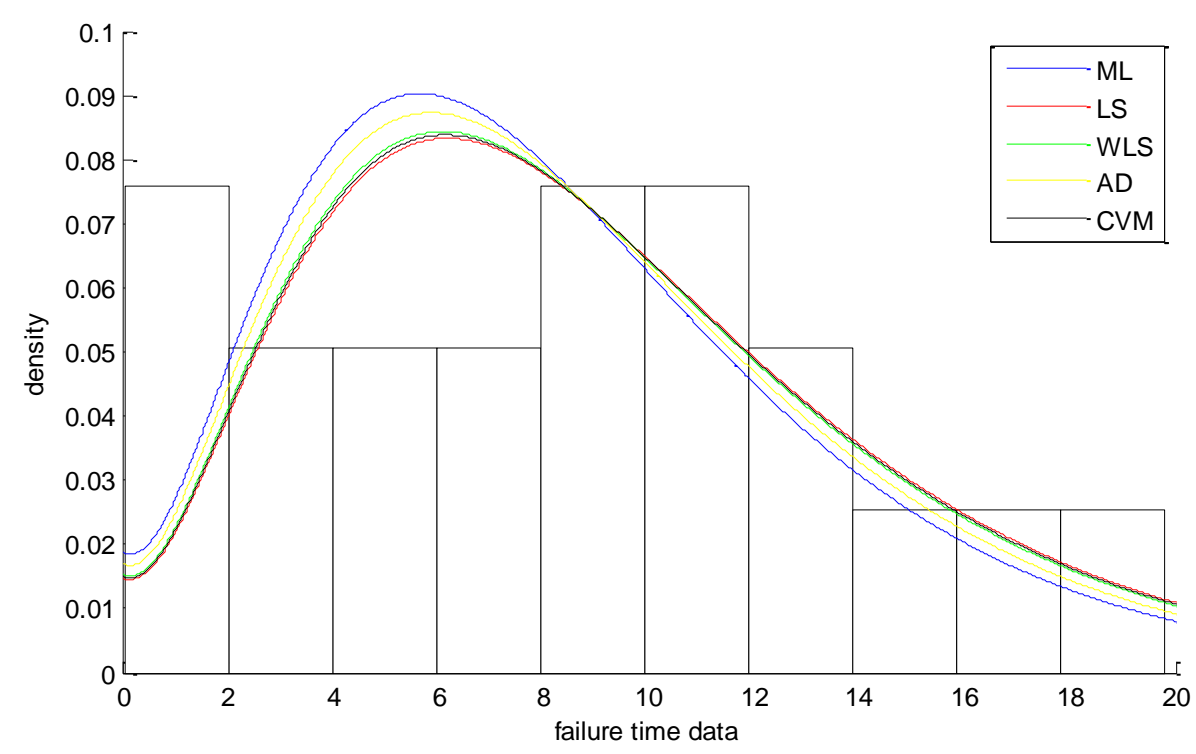

Figure 2. The density curves of Akash distribution for different methods of estimation based on failure time data

\section{Concluding remarks}

In this paper, the Akash distribution introduced by Shanker [1] is studied in terms of point estimations. Five estimators are discussed to estimate the parameter of Akash distribution. A new extension is provided for the estimation of the parameter of Akash distribution. Simulation studies are carried out for four different initial values. As the sample of size increases, it can be said that the MSEs and biases of all estimators decreases and close to each other. In a small sample, the MSEs of the MLE and ADE are smaller than the others. To show capability of Akash distribution in practical data modeling, a real data application is conducted. According to Table 3, it can be concluded that Akash distribution is the best fitting model for failure time data. Also, Figures 1-2 provides fitted cdfs and pdfs according to five different estimators for Akash distribution. The Akash distribution was compared with some known distributions and presented the estimates according to different parameter estimators.

\section{Conflicts of interest}

The authors declare that there is no conflict of interest.

\section{References}

[1] Amin K. Shanker R. Akash distribution and Its Applications, International Journal of Probability and Statistics, 4 (3) (2015) 65-75.
[2] Shanker R., Hagos, F. On modeling of lifetime data using Akash, Shanker, Lindley and exponential distributions, Biometrics \& Biostatistics International Journal, 3(6) (2016) 214-224.

[3] Shanker R. Shanker distribution and its applications, International journal of statistics and Applications, 5(6) (2015) 338-348.

[4] Lindley D.V. Fiducial distributions and Bayes' Theorem, Journal of the Royal Statistical Society, 20(1) (1958) 102-107.

[5] Shanker R., Shukla K. K. On two-parameter Akash distribution, Biom. Biostat. Int. J, 6(5) (2017) 416-425.

[6] Shanker R., Shukla K. K., Shanker R., Pratap, A. A generalized akash distribution, Biometric \& Biostatistics International Journal, 7(1) (2018) 18-26.

[7] Shanker R., Shukla K. K., Leonida T. A. A TwoParameter Poisson-Akash Distribution with Properties and Applications, International Journal of Probability and Statistics, 7(4) (2018) 114-123.

[8] Ganaie R. A., Rajagopalan, V. On Weighted Three Parameters Akash Distribution with Application of Lifetime data, Journal of Xidian University, 14 (4) (2020) 877-893.

[9] Gupta R. D., Kundu D. Generalized exponential distribution: different method of estimations, Journal of Statistical Computation and Simulation, 69(4) (2001) 315-337. 
[10] Kundu D., Raqab M. Z. Generalized Rayleigh distribution: different methods of estimations, Computational statistics \& data analysis, 49(1) (2005) 187-200.

[11] Mazucheli J., Louzada F., Ghitany M. E. Comparison of estimation methods for the parameters of the weighted Lindley distribution, Applied Mathematics and Computation, 220 (2013) 463-471.

[12] Dey S., Dey T., Kundu D. Two-parameter Rayleigh distribution: different methods of estimation, American Journal of Mathematical and Management Sciences, 33(1) (2014) 55-74.

[13] Mohammadi K., Alavi O., Mostafaeipour A., Goudarzi N., Jalilvand M. Assessing different parameters estimation methods of Weibull distribution to compute wind power density, Energy Conversion and Management, 108 (2016) 322-335.

[14] Dey S., Zhang C., Asgharzadeh A., Ghorbannezhad M. Comparisons of methods of estimation for the NH distribution, Annals of Data Science, 4(4) (2017) 441-455.

[15] Shanker R. Sujatha distribution and its applications, Statistics in Transition-New series, 17(3) (2016) 391-410.

[16] Murthy D. N. P., Xie M., Jiang R. Weibull Models, New York: Wiley, (2004). 